\title{
Design and Assembly Technology for the thermal insulation of the W7-X cryostat
}

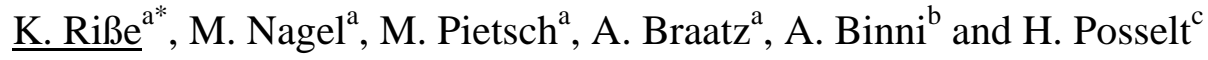 \\ ${ }^{a}$ Max-Planck-Institut für Plasmaphysik (IPP), Euratom Association, Wendelsteinstraße 1, D-17491 Greifswald \\ ${ }^{b}$ MAN Diesel \& Turbo SE, Dpt. OSA, Werftstraße 17, D-94469 Deggendorf, Germany \\ ${ }^{c}$ Linde AG Engineering Div., Dr.-Carl-von-Linde-Straße 6-14, D-82049 Hoellriegelskreuth
}

The Max-Planck-Institut für Plasmaphysik in Greifswald is building up the stellarator fusion experiment Wendelstein 7-X (W7-X). To operate the superconducting magnet system the vacuum and the cold structures are protected by a thermal insulated cryostat. The plasma vessel forms the inner cryostat wall, the outer wall is realised by a thermal insulated outer vessel. In addition 254 thermal insulated ports are fed through the cryogenic vacuum to allow the access to the plasma vessel for heating systems, supply lines or plasma diagnostics.

The thermal insulation is being manufactured and assembled by MAN Diesel \& Turbo SE (Germany). It consists of a multi-layer insulation (MLI) made of aluminized Kapton with a silk like fiberglass spacer and a thermal shield covering the inner cryostat surfaces. The shield on the plasma vessel is made of fibreglass reinforced epoxy resin with integrated copper meshes. The outer vessel insulation is made of brass panels with an average size of $3.3 \times 2.0 \mathrm{~m}^{2}$. Cooling loops made of stainless steel are connected via copper strips to the brass panels. Especially the complex $3 \mathrm{D}$ shape of the plasma vessel, the restricted space inside the cryostat and the consideration of the operational component movements influenced the design work heavily. The manufacturing and the assembly has to fulfil stringent geometrical tolerances e.g. for the outer vessel panels $+3 /-2 \mathrm{~mm}$.

Keywords: thermal insulation, cryostat, stellarator, Wendelstein 7-X

\section{Introduction}

The superconducting magnet system of the Wendelstein 7-X (W7-X) stellarator is surrounded by a thermal insulated cryostat. The plasma vessel with a surface of app. 190 sq.m forms the inner cryostat wall, the outer wall is realised by a thermal insulated outer vessel with a surface of app. 430 sq.m (see Fig. 1). In addition 254 thermal insulated ports are fed through the cryogenic vacuum to allow the access to the plasma vessel for heating systems, supply lines or plasma diagnostics. The port surface is app. 330 sq.m. The W7$\mathrm{X}$ is divided in 5 equal modules. The thermal insulation against ambient temperature outside and the plasma vessel temperature inside is provided by high vacuum in combination with a thermal insulation with active cooled shield on the components. The design criteria for the heat loss of the whole cryostat insulation is $<9 \mathrm{~kW}$ if the temperature difference from the cold to the warm cryostat components is about $290 \mathrm{~K}$. [1,2,3]

The thermal insulation is being manufactured and assembled by MAN Diesel \& Turbo SE (Germany). The development process and the detailed engineering of the thermal insulation were accompanied by the subcontractor Linde AG (Germany). The thermal insulation of the outer cryostat wall and the ports consists of a multi-layer insulation (MLI) made of aluminized Kapton with a silk like fibreglass spacer and a thermal shield made of brass covering the inner surfaces facing the cold magnet system . The shield on the plasma vessel is made of fibreglass reinforced epoxy resin with integrated copper meshes.

\section{Design of thermal insulation}

\subsection{Outer cryostat vessel insulation}

The outer vessel of one module is structured into a lower and an upper half shell. The outer vessel insulation is made of actively cooled brass panels combined with a MLI. Cooling loops made of stainless steel are connected via copper strips to the brass panels.

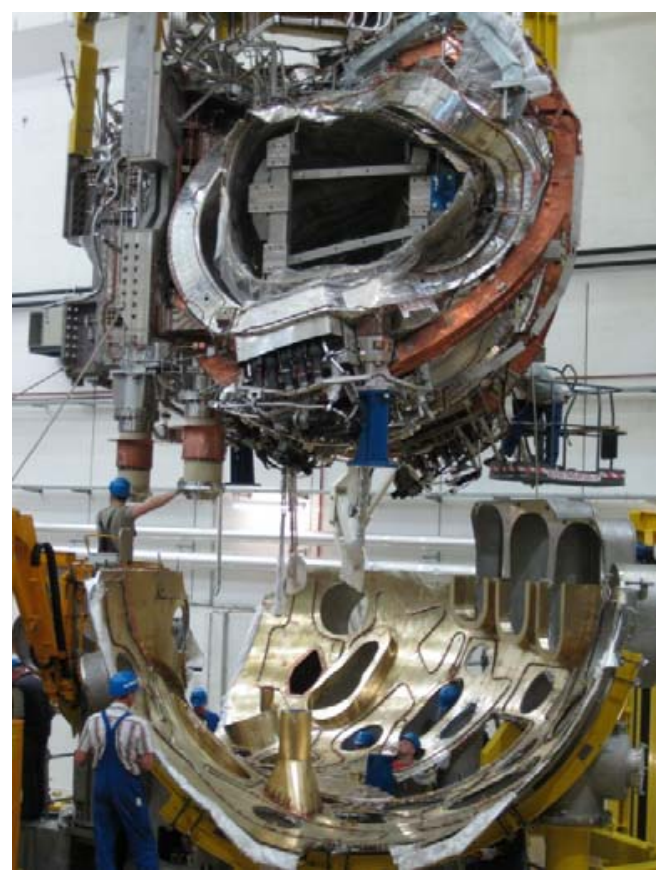

Fig.1 Assembly of one magnet module into the thermal insulated lower outer vessel half shell. 
The cooling pipes are cooled by He gas which keep the shield at $80 \mathrm{~K}$ during steady cryogenic conditions. Eleven panels with an average size of 3.3 x 2.0 sq.m are needed for the thermal insulation of one outer vessel half shell (see Fig. 3 and 5). The panels have a lot of circular openings due to a high number of openings for ports or for access to the cryostat. For the design it was determined that the outer vessel shield is assembled on nominal CAD level. The +/- 22mm manufacturing tolerances of the outer vessel can be adjusted by an individual length of the Torlon spacers. These individual Torlon spacers keep the brass shield in the nominal CAD position (see Fig.2).

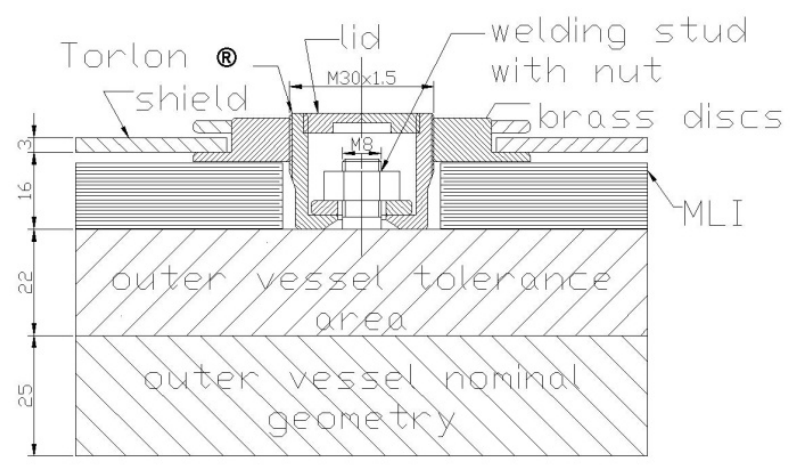

Fig.2 Cross section of thermal insulated outer vessel

Depending from the manufacturing tolerance of the outer vessel; the distance between the vessel and the shield can differ from 16 to $58 \mathrm{~mm}$. The Torlon spacers are screwed on thread bolts which are welded to the vessel (see Fig.2). The position of the thread bolts will be determined by a 3D measurement process. After the geometry evaluation the individual length of the Torlon spacers is defined.

The space between the vessel wall and the shield is filled with 20 layers of a multi-layer insulation (MLI, see Fig.2). The MLI is made of aluminized Kapton foil with a silk like fibreglass spacer layer. To achieve a precise form of the 3mm thick brass panels, the form are cut out on a CNC- controlled water jet cutting machine.

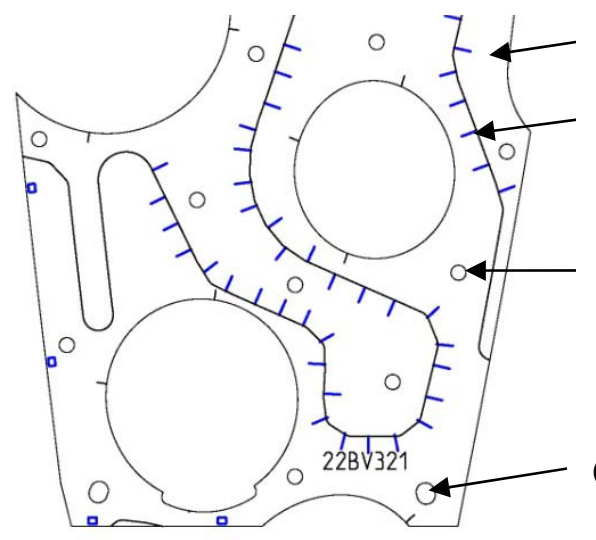

Fig.3 CAD Model of one outer vessel panel: brass shield (1), cooling pipes thermally connected to the brass shield (2), Torlon spacers as non-locating bearings (3) or fixed bearings (4)
The cooling pipe routing is marked on the panel surface which allows an easier assembly of the pre bended pipes. The pipes are pre-tinned which allows the soldering of high conductive copper stripes to the pipes. The copper stripes are welded to the brass panels.

The transition of the MLI on the panel border to the neighboring MLI is done in two steps to avoid a shining through effect. At first the MLI of one panel has an supernatant of $25 \mathrm{~mm}$ for the one half of the MLI layers and $55 \mathrm{~mm}$ for the second half of the layers, while the MLI of the corresponding partner panel is shortened and allows to push the MLI from one panel underneath the other panel. In $10 \mathrm{~mm}$ gap between the brass panels is covered with a thin bronze plate and supported by stainless steel stabilizers. To keep the MLI in position during assembly the MLI is hold by temporary label holders. [3]

A very comprehensive collision control process was established in the W7-X project to cope the very limited space inside the cryostat. [4] A tolerance chain is defined for each cryostat component which considers tolerances for manufacturing and assembly. In addition the component movement during cool down or during current operation is considered. These collision controls influence the design e.g. the outer vessel shield got corrugations or the shield pipe routing is influenced by narrowing components. The Fig. 4 visualizes the tolerances for the pipes on the outer vessel shield.

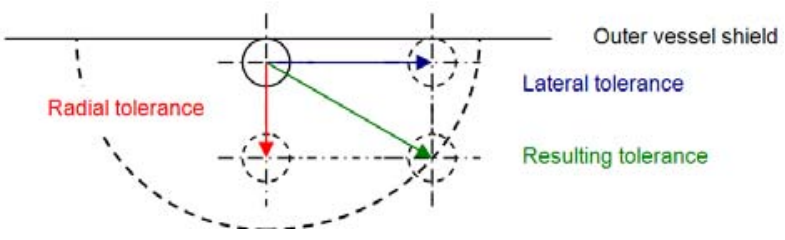

Fig.4 Cooling pipe tolerances

For the pipes a lateral tolerance for manufacturing is given with $2.5 \mathrm{~mm}$ and for assembly with $5.5 \mathrm{~mm}$; the total lateral tolerance is the sum of both values. At the end of the assembly process the two outer vessel shells are aligned to each other. At this stage the position of all components is proofed by a 3D laser tracker survey.

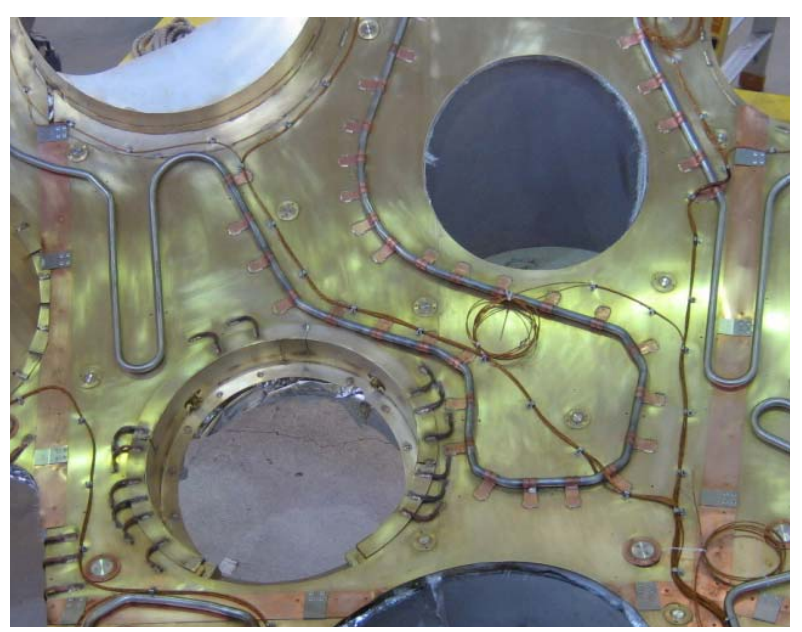

Fig.5 Final assembled outer vessel panel with the same view like Fig. 3 


\subsection{Plasma vessel insulation}

The plasma vessel of the W7-X machine has a complex 3D shape which is determined by the helical magnetic field of the W7-X stellarator. The maximum temperature difference occurs during the baking phase of the plasma vessel at $150^{\circ} \mathrm{C}$ whereas the plasma vessel shield is cooled to $80 \mathrm{~K}$. The structure of the thermal insulation of the plasma vessel is similar to the outer vessel with the deviation that instead of the brass shield a glass fibre shield with integrated copper mash mats were used. The fibre shields were laminated on special forms; the three copper mats were integrated into the fibre compound. The copper mats distribute the heat inside the fibre panels and transfer the heat to the cooling pipes. The cooling pipes are thermally contacted to the fibre shield by copper braids (see Fig. 6). Copper clamps that are connected to the copper braids are soldered to the cooling pipes and on the other end the tin coated copper braids are riveted to the fibre panels with a contact surface of app. 150 sq. mm each.

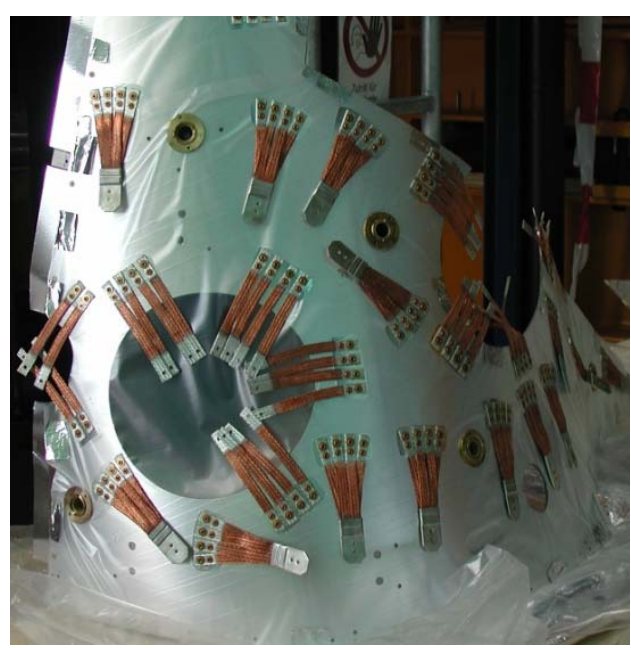

Fig.6 Copper braids on the plasma vessel shield

\subsection{Port insulation}

The W7-X machine has 254 ports which are fed through the cryogenic vacuum to allow the access to the plasma vessel for heating systems, supply lines or plasma diagnostics. Adapted to the function and the available space in the modules the ports have a different size and different shapes.

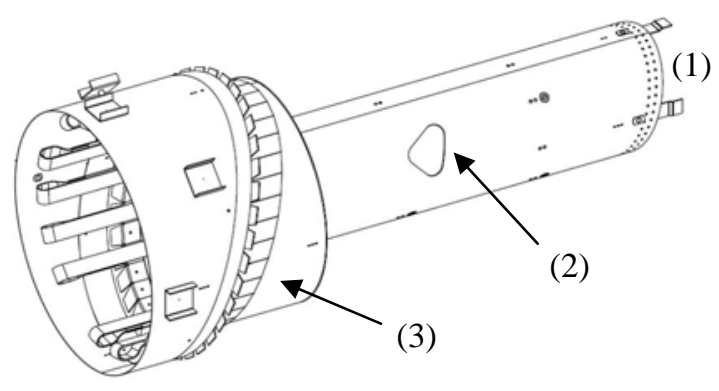

Fig.7 Final assembly sketch of a port shield; (1) plates for plasma vessel fixation; (2) pipe shield with cut outs, (3) port bellow insulation with fixation and thermal contact to outer vessel shield

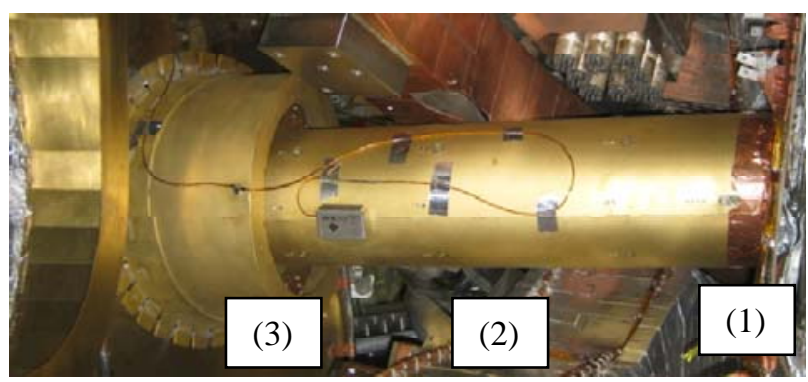

Fig.8 Installed port with thermal insulation; numbers (1) (2) (3) equal to Fig. 7

The port itself is welded to the plasma vessel and the outer vessel. The structure of the thermal insulation consist of an outer $2 \mathrm{~mm}$ thick brass shield, 20 layers of MLI and on the inner side a $0.3 \mathrm{~mm}$ thick bronze sheet gives mechanical support (see Fig. 7 and 8). The bronze sheet allows a relative displacing of the port body to the thermal insulation during assembly. The thermal shield is mechanically fixed to the plasma vessel and supported by the stainless steel port itself. The port MLI has an overlap with the plasma vessel MLI. On the opposite end the port shield is fixed and contacted to the outer vessel shield. The brass shield is cooled by thermal conductivity through the contact to the outer vessel or plasma vessel shields. During port assembly the welding to the plasma vessel is protected by protective gas which is leaded in a Teflon tube inside the MLI.

\subsection{Other components}

Several other components need to be thermal insulated e.g. heating pipes on the plasma vessel which are routed through the cryostat to the outer vessel wall, the plasma vessel supports and the so called cryo legs, which are the main supports of the cold mass. [5]

\section{Assembly Process}

The W7-X machine is structured in 5 equal modules. The outer vessel has five module segments and each vessel module consists of a lower and an upper half shell. These large steel components have an allowed tolerance of $+/-22 \mathrm{~mm}$ [6]. The assembly of the thermal insulation for the outer vessel is done by the MAN Company with following sequence: (1.) Optimal alignment of the lower and upper half shell to the nominal CAD contour and assembly of the shells into a heavy support frame. (2.) Trial Assembly of the upper to the lower shell. A geometrical survey with a laser tracker system is done to determine the position of the Torlon holders and the individual Torlon holder length. (3.) Separation of half shells for assembly. (4.) Installation of pre manufactured dome shield insulation and start of panel installation. (5.) Precise assembly of the cooling pipes to the panel surface. (6.) Soldering of copper stripes on the pipes for thermal connection to the brass shield. (7.) Assembly of the half shells to each other for final 3D survey. The final 3D report has to proof that the assembly tolerances are fulfilled. The report e.g. for the fourth module contains the check for 22 panels (see Fig.9) with 24 corrugations, $160 \mathrm{~m}$ cooling pipes, 28 dom shields and 9 thermal sensors. 


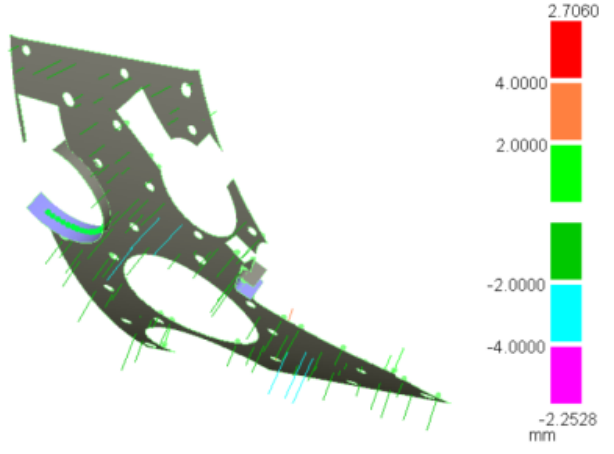

Fig.9 Results from the 3D survey of one shield panel with deviation vectors between $+2.7 \mathrm{~mm}$ and $-2.2 \mathrm{~mm}$

The ports will be assembled when the thermal port insulation is already installed. The insulated port is fixed to an assembly ramp. This ramp can drive the port on a guidance line to the final position in the cryostat. The ramp allows a precise handling of the port which can weigh up to $1000 \mathrm{~kg}$. The ramp can work from the basement; can be installed on an inner or outer tower or on a bridge between the two towers to allow the port assembly from the top (see Fig.10). The assembly is structured into two steps. In the first step the insulation of the port bellow is mounted to the outer vessel. In a second step the whole insulated port is moved into the cryostat. During an intermediate step the port shield will be fixed on the plasma vessel and will be thermally connected to the outer vessel shield and the plasma vessel shield. Then the port moves into the final position where the welding to the plasma vessel and outer vessel will take place.

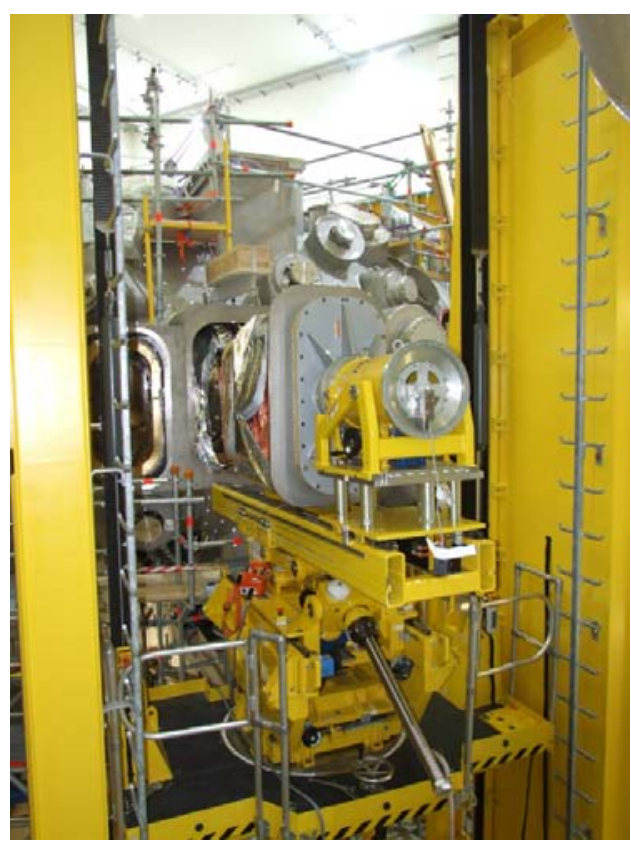

Fig.10 Large thermal insulated port fixed on the ramp. The ramp operates from a platform on the outer tower.

\section{Manufacturing and Assembly Progress}

The design, manufacturing and assembly of the thermal insulation are well advanced. All five plasma vessel modules are thermally insulated. The outer vessel insulation is finished on 3 of 5 modules. The port assembly has been started in March 2010 on the first module. To fit the port to the plasma and to the outer vessel needs more time than planned before.

It turned out that the whole process is heavily influenced by the complex 3D geometry and the efforts to avoid collisions. The thermal shield components became a more complex 3D surface which increased the efforts for design and manufacturing. Countermeasures were taken to reduce these efforts e.g. it was succeeded to define port shield as series components and assembly tolerances for cryogenic pipes were relaxed. The thermal insulation of the module separation plane as last working package did not started yet.

\section{Summary}

The main components of the W7-X cryostat are the plasma vessel, the outer vessel and the ports. The thermal insulation of the plasma vessel is finished. The insulation of the outer vessel is installed on 3 of 5 modules. The design work, the manufacturing and the assembly of the port insulation is ongoing. The most difficult challenges during the design were the implementation of a comprehensive collision control process with related design adaptations. The manufacturing process of the components runs well with increased efforts to fulfil the geometrical tolerances. The port installation is hindered by difficulties to fit and adjust the steel of the ports to the plasma vessel geometry. Extensive 3D surveys accompany the manufacturing and assembly process.

\section{Acknowledgments}

The collaboration for the design; the manufacturing and the assembly of the thermal insulation with the companies MAN and Linde AG is gratefully acknowledged. Sincere thanks to all my colleagues from the W7-X team for their support and contributions to this paper.

\section{References}

[1] L. Wegener, "Status of Wendelstein 7-X Construction," Fusion Engineering and Design, 84 (2009), pp. 106-112.

[2] H.-S. Bosch, Wendelstein 7-X a technology step toward Demo, IEEE Transactions on Plasma Science, Vol.38, No3, March 2010.

[3] M. Nagel, "Thermal and Mechanical Analysis on Wendelstein 7-X Thermal Shield”, 26th Soft 2010

[4] Ch. Baylard, "Configuration space control for Wendelstein 7-X” Fusion Engineering and Design, 84 (2009), pp. 435-440

[5] T. Koppe, "Overview of main-mechanical-components and critical manufacturing aspects of the Wendelstein 7 X cryostat", 26 $6^{\text {th }}$ Soft 2010

[6] B. Hein, "Manufacturing and Assembly of the Plasmaand Outer Vessel of the cryostat for Wendelstein 7-X", $26^{\text {th }}$ Soft 2010 